\title{
Integration of epidemiological findings with mechanistic evidence in regulatory pesticide risk assessment: EFSA experiences
}

Pelkonen, Olavi; Bennekou, Susanne Hougaard; Crivellente, Federica; Terron, Andrea; Hernandez, Antonio F.

Published in:

Archives of Toxicology

Link to article, DOI:

10.1007/s00204-019-02467-w

Publication date:

2019

Document Version

Peer reviewed version

Link back to DTU Orbit

Citation (APA):

Pelkonen, O., Bennekou, S. H., Crivellente, F., Terron, A., \& Hernandez, A. F. (2019). Integration of epidemiological findings with mechanistic evidence in regulatory pesticide risk assessment: EFSA experiences. Archives of Toxicology, 93(6), 1779-1788. https://doi.org/10.1007/s00204-019-02467-w

\section{General rights}

Copyright and moral rights for the publications made accessible in the public portal are retained by the authors and/or other copyright owners and it is a condition of accessing publications that users recognise and abide by the legal requirements associated with these rights.

- Users may download and print one copy of any publication from the public portal for the purpose of private study or research.

- You may not further distribute the material or use it for any profit-making activity or commercial gain

- You may freely distribute the URL identifying the publication in the public portal 


\section{Archives of Toxicology \\ Integration of epidemiological findings with mechanistic evidence in regulatory pesticide risk assessment - EFSA experiences

\author{
--Manuscript Draft--
}

\begin{tabular}{|c|c|}
\hline Manuscript Number: & \\
\hline Full Title: & $\begin{array}{l}\text { Integration of epidemiological findings with mechanistic evidence in regulatory } \\
\text { pesticide risk assessment - EFSA experiences }\end{array}$ \\
\hline Article Type: & Meeting Report \\
\hline Corresponding Author: & $\begin{array}{l}\text { Olavi Pelkonen } \\
\text { University of Oulu } \\
\text { Oulu, FINLAND }\end{array}$ \\
\hline $\begin{array}{l}\text { Corresponding Author Secondary } \\
\text { Information: }\end{array}$ & \\
\hline Corresponding Author's Institution: & University of Oulu \\
\hline $\begin{array}{l}\text { Corresponding Author's Secondary } \\
\text { Institution: }\end{array}$ & \\
\hline First Author: & Olavi Pelkonen \\
\hline First Author Secondary Information: & \\
\hline Order of Authors: & Olavi Pelkonen \\
\hline & Susanne H Bennekou \\
\hline & Federica Crivellente \\
\hline & Andrea Terron \\
\hline & Antonio F Hernandez \\
\hline Order of Authors Secondary Informe & \\
\hline Funding Information: & \\
\hline Abstract: & $\begin{array}{l}\text { Toxicological risk assessment of plant protection products (PPP) is currently carried } \\
\text { out with the principal input from regulatory toxicology studies following OECD test } \\
\text { guidelines, with little input from epidemiological data. An EFSA-commissioned } \\
\text { systematic review of pesticide epidemiological studies (Ntzani et al 2013) revealed } \\
\text { statistically significant associations, among others, between pesticide exposures and } \\
\text { Parkinson's disease and childhood leukaemia. Thereafter, EFSA launched a project } \\
\text { with a mandate for the Plant Protection Products and their residues (PPR) Panel to set } \\
\text { the ground for the use of epidemiological data in the risk assessment of pesticides, as } \\
\text { requested by Regulation (EC) } 1107 / 2009 \text {. The project culminated with the publication } \\
\text { of two EFSA's scientific opinions on the potential contribution of experimental } \\
\text { investigations and epidemiological studies in PPP risk assessment and with the } \\
\text { scientific conference held on } 20 \text { November, 2017, in Parma, Italy. The application of } \\
\text { modern methodologies in exposure assessment, toxicology and epidemiology would } \\
\text { improve the pesticide risk assessment process and support a mechanistic shift for the } \\
\text { integration of these three disciplines under a novel paradigm in risk assessment. The } \\
\text { application of the Adverse Outcome Pathways (AOP) conceptual framework to this } \\
\text { approach would contribute to gain insight into the biological plausibility of a hazard } \\
\text { identified in epidemiological or experimental studies and would inform an Integrated } \\
\text { Approach to Testing and Assessment (IATA) within a regulatory context. }\end{array}$ \\
\hline Suggested Reviewers: & \\
\hline
\end{tabular}


Final Version 190329

Meeting $\operatorname{Report}^{1}$

Integration of epidemiological findings with mechanistic evidence in regulatory pesticide risk assessment - EFSA experiences

\author{
Olavi Pelkonen ${ }^{1}$, Susanne H. Bennekou ${ }^{2}$, Federica Crivellente ${ }^{3}$, Andrea Terron ${ }^{3}$, Antonio F. \\ Hernande $z^{4}$ \\ ${ }^{1}$ University of Oulu, Research Unit of Biomedicine/Pharmacology and Toxicology, Oulu, \\ Finland \\ ${ }^{2}$ National Food Institute, Division for Risk Assessment and Nutrition, Technical University of \\ Denmark \\ ${ }^{3}$ EFSA, European Food Safety Authority, Parma, Italy \\ ${ }^{4}$ University of Granada School of Medicine, Granada, Spain
}

Correspondence:

Olavi Pelkonen, University of Oulu, Research Unit of Biomedicine/Pharmacology and Toxicology, Aapistie 5 B, FIN-90014 Oulu (Finland); e-mail: olavi.pelkonen@ oulu.fi; mobile: +358405075196

\footnotetext{
${ }^{1}$ This report is based on plenary talks and extensive discussions at the EFSA Scientific conference on the use of epidemiological findings in regulatory pesticide risk assessment on 21 November, 2017, in Parma, Italy. The speakers were Federica Crivellente (EFSA), Susanne Hougaard Bennekou (Danish EPA, EFSA PPR Panel), Bette Meek (University of Ottawa), Antonio Hernandez Jerez (University of Granada, EFSA PPR Panel), David Miller (US-EPA), Karin Angeli (ANSES), Laura Beane Freeman (US NCI), Judy Lakind (LaKind Associates), Marie-Odile Rambourg (ANSES), Manolis Kogevinas (ISGlobal), Carol Burns (ECPA), Martin Dermine (PAN Europe). The speakers and discussants are thanked for their contributions during the meeting; however, report authors are responsible for views and recommendations expressed in this report (see the EFSA site: https://www.efsa.europa.eu/en/events/event/171121-0.
} 


\section{Abstract}

Toxicological risk assessment of plant protection products (PPP) is currently carried out with the principal input from regulatory toxicology studies following OECD test guidelines, with little input from epidemiological data. An EFSA-commissioned systematic review of pesticide epidemiological studies (Ntzani et al 2013) revealed statistically significant associations, among others, between pesticide exposures and Parkinson's disease and childhood leukaemia. Thereafter, EFSA launched a project with a mandate for the Plant Protection Products and their residues (PPR) Panel to set the ground for the use of epidemiological data in the risk assessment of pesticides, as requested by Regulation (EC) 1107/2009. The project culminated with the publication of two EFSA's scientific opinions on the potential contribution of experimental investigations and epidemiological studies in PPP risk assessment and with the scientific conference held on 20 November, 2017, in Parma, Italy. The application of modern methodologies in exposure assessment, toxicology and epidemiology would improve the pesticide risk assessment process and support a mechanistic shift for the integration of these three disciplines under a novel paradigm in risk assessment. The application of the Adverse Outcome Pathways (AOP) conceptual framework to this approach would contribute to gain insight into the biological plausibility of a hazard identified in epidemiological or experimental studies and would inform an Integrated Approach to Testing and Assessment (IATA) within a regulatory context.

Key words: plant protection products, pesticides, risk assessment, epidemiology, adverse outcome pathway (AOP), exposure, Integrated Approach to Testing and Assessment (IATA) 


\section{Introduction}

According to the current European Union (EU) legislation on the placing of plant protection products (PPP) on the market, epidemiological studies are of particular value and must be submitted 'where available, and supported with data on levels and duration of exposure, and conducted in accordance with recognised standards' (Regulation No. 1107/2009). Likewise, Regulation No. 283/2013, concerning the data requirements for active substances (AS), laid down that 'relevant epidemiological studies shall be submitted, where available', but there is 'no obligation for the petitioners to conduct epidemiological studies for the AS undergoing the approval or renewal process'. However, a systematic literature review is required for the AS and its relevant metabolites, although this is not restricted to human observational studies but should also include experimental studies published in the open literature.

Epidemiological data on a specific pesticide exposure has not been submitted to the regulatory authority at first approval and only occasionally they are provided at the time of renewal of an AS and consequently has rarely contributed to the risk assessment process thus far. Nevertheless, several epidemiological studies and metanalyses are available in the scientific literature; though despite the large amount of epidemiological studies reporting associations between pesticide exposure and human health outcomes, the impact of such studies in regulatory risk assessment is still limited.

EFSA commissioned to University of Ioannina (Greece) a systematic literature review and meta-analyses of epidemiological studies published from 2006 to 2012 for surveying potential associations between pesticide exposure and a wide array of human adverse health outcomes (Ntzani et al 2013). Although statistically significant associations were found for some diseases (liver cancer, breast cancer, stomach cancer, amyotrophic lateral sclerosis, asthma, type II diabetes, childhood leukaemia and Parkinson's disease), no firm conclusions could be drawn for the majority of them. Furthermore, the report alluded that the epidemiological studies reviewed suffered from a number of limitations and large heterogeneity of data, including broad pesticides definitions (and therefore inaccurate pesticide exposure estimates) and consequently the scope of the report did not allow drawing in-depth associations between pesticide exposure and specific health outcomes.

On the basis of the external report, in 2013 EFSA initiated a Pesticide Epidemiology project, which started with a "Stakeholder Workshop on the use of epidemiological findings in regulatory pesticide risk assessment" held on 18 February 2015 in Paris. The project 
culminated four years later in the publication of two scientific opinions: Scientific Opinion on the Investigation into Experimental Toxicological Properties of plant protection products (PPPs) Having a Potential Link to Parkinson's Disease and Childhood Leukaemia (EFSA PPR Panel, 2017a), and Scientific Opinion of the PPR Panel on the follow-up of the findings of the External Scientific Report "Literature review of epidemiological studies linking exposure to pesticides and health effects" (EFSA PPR Panel, 2017b). The two Scientific Opinion were also presented and debated at the EFSA Conference on epidemiology on 20 November, 2017, in Parma, Italy. Additional initiatives are currently ongoing in EFSA with the recognition that epidemiology is an overarching item for EFSA and as such will be led by the Scientific Committee.

This paper aims to raise awareness of the scientific community about this initiative by summarizing the results and recommendations of the above mentioned EFSA project, i.e. search ways to integrate experimental, epidemiological and regulatory approaches for pesticide risk assessment. Additional details of the project can be found at the EFSA website (https://www.efsa.europa.eu/en/news/62081). A scheme of the project is presented in Figure 1.

\section{Epidemiological studies - role in pesticide risk assessment and room for improvements}

The Scientific Opinion (EFSA PPR Panel, 2017b) proposed a methodological approach specific for pesticide ASs to make appropriate use of epidemiological data for risk assessment. The approach should include the analysis of strengths and weaknesses of epidemiological studies after the appropriate quality considerations as well as the investigation of biological plausibility of the epidemiological associations (Figure 2).

\section{Major limitations in current pesticide epidemiological studies}

The systematic appraise of the epidemiological evidence allows a number of methodological limitations to be identified. These limitations prevent robust conclusions to be drawn, and they include, but are not limited to: a) less than optimal study designs, as most of studies are case-control and cross-sectional studies, which lack temporal concordance. Besides, many studies are not sufficiently powered; b) use of broad definition of exposure assessed through questionnaires (often not validated) and seldom by biomarkers of exposure in biological matrices. Besides, information on exposure to individual pesticides is scarce and, where available, very often it is not quantitatively reported; c) deficiencies in outcome assessment 
(broad outcome definitions and use of self-reported outcomes or surrogate outcomes); d) deficiencies in reporting, confounder control and statistical analysis (including multiple testing); f) selective reporting of results and publication bias.

\section{Pesticide exposure data in environmental epidemiology: limitations and quality assessments}

There are large methodological difficulties in assessing and measuring exposure to pesticides in relation to epidemiological investigations. Human pesticide exposures are most of the time complex, involving many active substances, co-formulants and other ingredients. Exposures can be occupational (applicators and farmworkers), para-occupational (by-standers) or residential; and may be acute (as a result of exposure to high doses), or chronic, that is longterm low-dose (as a result of intermittent, irregular, but usually highly variable exposure with respect to time and intensity). Pesticide exposure may be measured by environmental analyses, personal exposure monitoring or by monitoring of human material (e.g., blood, urine, hair...). Alternatively, exposure can be modelled using job-exposure or crop-exposure matrices, geo-coding residential addresses, etc. Because of all these complexities, exposure misclassification occurs to a large extent in pesticide epidemiology such that the possibility to detect an adverse effect associated to a specific AS is less likely. Preferentially exposure assessments should be designed for specific situations depending on specific hypotheses, outcomes, timing, intensity etc. In any case, improvements in exposure assessment are critically important because there is relatively convincing knowledge about the health risk effects of pesticide exposures.

Because of the increasing interest in using epidemiology for regulatory decision-making, there is obviously a growing demand for high quality exposure data. Methodological limitations of individual studies make meaningful weight of the evidence assessment difficult. Transparent and systematic instruments are in demand for use for both study design and quality assessment, as well as to help to address problems in exposure assessment. One such tool is Biomonitoring, Environmental Epidemiology, and Short-lived Chemicals (BEES-C) instrument (LaKind et al 2014: Goodman et al 2017), which is in use in USA and Europe. Exposure quality evaluation according to this instrument regarding especially biomarker development includes the following elements: exposure and biological relevance, specificity, method sensitivity, contamination, stability, method requirements, adjust for matrix dilution, temporality, variability/misclassification, and general epidemiological study design considerations. A recent review stresses the importance of multiple biomonitoring samples 
collected over a period of toxicological relevance and with consideration of exposure patterns (LaKind et al 2019).

\section{Reliability and relevance of epidemiological studies}

The Scientific Opinion (EFSA PPR Panel, 2017b) also focused on matters which would enhance the quality and relevance of epidemiological research on pesticides for risk assessment purposes, such as $1 /$ adequate assessment of exposure at individual pesticide level to minimize exposure misclassification, 2/ valid and reliable outcome assessment, 3/ accounting for potential confounders and 4/ adequate statistical analysis and reporting of results. Furthermore, systematic reviews and meta-analyses of the available epidemiological evidence can provide a useful approach for hazard identification as these tools allow generation of summary data, increase the statistical power and precision of risk estimates by combining the results of all individual studies (e,g, Moher et al, 2015; Shamseer et al, 2015). The crucial goal is the integration of epidemiological and toxicological data in the process of hazard identification/characterization and weighting the evidence from different sources, e.g. observational, in vivo, in vitro and in silico studies (Hernandez and Tsatsakis, 2017). The reliability, relevance and consistency of single studies and pooled evidence should be considered for a weight of evidence approach. This, together with all available data, will be used in an integrated approach to testing and assessment (IATA) where the available mechanistic data will lend support to the development of appropriate Adverse Outcome Pathway (AOP); AOP informed IATA will therefore contribute to pesticide risk assessment. Conclusions as to the role of epidemiological evidence in pesticide risk assessment included the following points: 1/ Current epidemiological studies can be useful for hazard identification/characterization of pesticides. 2/ Better designed epidemiological studies may improve risk assessment of pesticides. $3 /$ In this connection, it is important to stress that the assessment of exposure represents one of the most relevant limitations with the epidemiological studies carried out with pesticides. 4/ Biological/mechanistic plausibility supports associations between pesticide exposure and the adverse outcomes described in human epidemiological studies, including complex diseases that are unlikely captured by invivo experimental toxicological studies. 5/ AOP and mode of action (MoA) frameworks should be used to link the outcome from epidemiological studies in order to weight their conclusions and establish a mechanistic biologically plausible link between the AO and the experimental studies, and finally, 6/ integration of all these scientific evidence in a structured dose and temporal concordant framework would benefit from moving to a mechanistic-based 
risk assessment able to contribute to the identification of risk factors relevant to human diseases.

A point of disagreement among some stakeholders has been a question of all-inclusiveness of studies to assessment vs. quality-assessment of epidemiological evidence. Scientifically it is clear that quality of studies is a major determinant and it is not possible to base regulatory decisions on poor epidemiology data. Even good epidemiological studies may have very limited or no weight in the final assessment, if appropriate data is lacking or insufficient (e.g. data on specific pesticides under assessment). Still, with quality epidemiological studies important questions remain to be considered, for example: 1/ How can the regulatory process ensure optimal timing between the re-assessment process of AS and the availability of appropriate epidemiological studies, 2/ What is the relevance of negative results in risk assessment? (which relates to transparency of reporting the results), and 3/ Can dose-response data from epidemiological studies, if available, be used to identify a point of departure level suitable for benchmark-dose modelling, 4/ What kind of findings will trigger the adoption of precautionary measures in risk assessment as stipulated by the regulation? These are all very valid questions which have to be dealt with when integrating the different lines of evidence, i.e. epidemiology with experimental research.

\section{The US- Environmental Protection Agency (EPA) approach}

In the USA, the Environmental Protection Agency/Office of Pesticide Programs (EPA/OPP) has the central role in pesticide risk assessment due to its regulatory mandate. EPA/OPP is increasingly considering on-going epidemiological studies, and the collection and use of incident data for pesticide risk assessment. The main regulatory tool for this purpose is the OPP Framework for incorporating epidemiology and incident data (US-EPA 2016). EPA/OPP intends to make increasing use of these data for human health risk assessment under the most scientifically robust and transparent way. The guiding principles of the framework include the use of epidemiology reviews in a tiered process in problem formulation, the identification of major factors that will inform risk assessment, and MoA/AOP Framework to identify key events along a causal pathway where different sources of information (from experimental to observational studies) can be organized and integrated. The key issues of the OPP Framework are the assessments of exposure, health outcomes, confounding, statistical analysis and risk of bias of individual epidemiological studies. OPP has adopted a tiered review assessment approach to fulfil its regulatory mandate and respond to emerging public health issues, manage program workload and prioritize potential risk issues that warrant systematic 
investigation. Under this approach, each tier considers the usefulness of the assessment for its intended purpose in order to ensure that the assessment produced is suitable and useful for informing the needed decisions. Overall, concepts in EPA/OPP framework are similar in many ways to EFSA's proposed framework, although also some differences exist because of the different legal requirements (EFSA PPR Panel, 2017b).

\section{Agricultural cohort studies as key sources of pesticide epidemiological evidence}

One of the main sources of pesticide epidemiological findings is the US Agricultural Health Study (AHS), a federally funded study that evaluates associations between pesticide exposures and cancer and other health outcomes. The main features of the AHS studies include a more informative study design (prospective cohort), improved exposure assessment (self-reported, but ascertained in multiple ways and algorithms developed), a considerable number of study subjects ( 57000 applicators, $~ 32000$ spouses), a more precise outcome assessment (cancer registries, others self-reported, but ascertained by medical reports), approach to etiologic analyses, and sub-studies for specific hypotheses. The project has already resulted in numerous publications and it will continue, in some cases updating previous findings on specific exposures and health outcomes (Andreotti et al., 2018). Further details could be found at the AHS internet site (Ref: www.aghealth.nih.gov).

AGRICOH is a consortium of agricultural cohort studies from five continents (e.g., AHS, French Agriculture and Cancer Study (AGRICAN), Cancer in the Norwegian Agricultural Population (CNAP), etc.) initiated by the US National Cancer Institute and the International Agency for Research on Cancer (IARC) in October 2010. The aim is to encourage and support data pooling to study disease-exposure associations that individual cohorts do not have sufficient statistical power to study. Cohorts participating in AGRICOH study involve health outcomes in relation to environmental and occupational exposures in agricultural settings (Ref; agricoh.iarc.fr).

\section{Contribution of vigilance data to the risk assessment of pesticides}

Vigilance (surveillance and monitoring) systems include foremost activities related to the detection, assessment, understanding and prevention of adverse events. Currently there are systems able to detect pesticide-related incidences, such as work-related disease surveillance systems, occupational disease registries, post-marketing surveillance programmes, nonspecific recording systems such as Poison Control Centres (PCCs), and EU alerting system on 
chemical hazards (RASCHEM). However, there is considerable heterogeneity within and

\section{Use of the AOP framework to improve the utilization of epidemiological findings for pesticide risk assessment}

The AOP concept is becoming a practical and pragmatic tool in toxicological research and regulatory risk assessment (Delrue et al 3024; Edwards et al 2016; Sakuratani et al 2018; Vinken 2018). The development of specific AOPs for parkinsonian motor symptoms and infant leukaemia as adverse outcomes was the principal objective of the EFSA PPR Panel to set the biological plausibility of the epidemiological associations found between exposure to 
pesticides and the risk of developing Parkinson's disease (PD), and infant and childhood leukemia (EFSA PPR Panel, 2017a). Both diseases are complex entities and the first decision was the use specific symptoms (motor disturbances for PD) or biologically distinct entities (infant leukaemia or childhood leukaemia) as starting adverse outcomes (AOs) to develop the corresponding AOPs.

It is also of importance to note that motor disturbances could basically be captured by existing guidelines as clinical signs in both the standard repeat dose studies (OECD TG 408) as well as those developed for the study of neurotoxicity in adult and young laboratory animals (OECD TG 424) and the guideline for developmental neurotoxicity (OECD TG 426). However, degeneration of dopaminergic neurons at substantia nigra is not specifically covered by these guideline studies. Likewise, infant/childhood leukaemia are difficult to capture by the current regulatory testing paradigm used for hazard identification of pesticides as this is not designed to detect the particular changes that occur only during early (pre- and postnatal) life stages and models do not involve 'a second hit' that has been captured in experimental studies. The only available study that covers these critical stages is the Extended One-Generation Reproductive Toxicity Study (OECD TG 443); however, this is relatively recent and has not been applied to most of the pesticides currently marketed. Furthermore, the protocol was not designed to cover carcinogenic endpoints and the power of the study is probably not sufficient.

In short, there is a strong expectation that AOP development would provide biological plausibility for epidemiological observations, enabling to identify important etiological factors for complex human outcomes and to develop clinically useful biomarkers and thus support the improvement of hazard and risk assessment.

On the basis of the EFSA scientific opinion on the use of epidemiological studies for pesticide risk assessment (EFSA PPR Panel, 2017b), the conclusions involved the following important points: 1) AOP framework contributes to hazard identification and characterisation and it is useful in regulatory risk assessment to explore whether an AO (e.g., those identified in any OECD TG) is biologically plausible or not. However, chemical specific risk assessment benefits from MoA and/or IATA framework. 2) The prototype AOPs developed for PD and infant leukaemia supported the epidemiological findings indicating that pesticides interacting with specific MIEs and triggering downstream key events (KE) are indeed risk factors for PD and infant leukaemia. 3) The AOP framework is an appropriate tool to understand whether chemical hazards relevant to such human diseases can be explored and detected in standard 


\footnotetext{
${ }^{2}$ Bal-Price A, Leist M, Schildknecht S, Tschudi-Monnet F, Paini A, Terron A (2018), "Adverse Outcome Pathway on Inhibition of the mitochondrial complex I of nigro-striatal neurons leading to parkinsonian motor deficits", OECD Series on Adverse Outcome Pathways, No. 7, OECD Publishing, Paris, https://doi.org/10.1787/b46c3c00en., and Bal-Price A, Leist M, Schildknecht S, Tschudi-Monnet F, Paini A, Terron A. Inhibition of the mitochondrial complex I of nigro-striatal neurons leads to parkinsonian motor deficits (AOP 3 ). Draft version under review. https://aopwiki.org/aops/3

${ }^{3}$ Viviani B. Adverse Outcome Pathway: Redox-cycling of a chemical initiated by electrons released by the mitochondrial respiratory chain leading to parkinsonian motor deficits. Toxicol Let 2016; 259S: S62
} 
opinion (2017a). Specific investigation of the substantia nigra and dopaminergic neuronsn as recommended in the EFSA opinion (2017a), was required. ${ }^{4}$

In the overall scenario of the applicability of the AOP framework for assessing causality of observations in epidemiological studies, the following considerations should be accounted for: distinction between AOP and chemical-specific MOA, evolution of Weight of Evidence (WoE)/Confidence considerations in MOA/AOP analysis, and implications for assessment of causality in epidemiological studies for regulatory application (Bhat et al 2017; Rhomberg et al 2013). As mentioned above, AOPs are well suited to consideration of biological plausibility for causation in epidemiological studies. Another crucial factor is the assessment of, and confidence in, experimental support for AOPs. Besides, it is of importance to keep in mind the distinction between confidence in a mechanistic pathway (AOPs) and replication of a human effect in animal studies as support for biological plausibility underpinning causation in epidemiological studies (Meek et al 2014). Obviously such an analysis has some implications regarding planning, conduct and assessment of epidemiological studies for regulatory application. These include the need for common "metrics" for exposure and outcome assessment (whose elements should be precisely defined), analysis of confidence, inclusion of appropriate elements into study designs and epidemiological training of researchers and assessors. All these are required to facilitate purpose specific regulatory application.

\section{Considerations and recommendations for future work}

On the basis of the above coverage of the EFSA project and scientific opinions as well as additional considerations about strengths and limitations of pesticide epidemiological studies, it is opportune to summarize the current situation as to what will be the most important future needs and topics in research and development as follows:

\section{Assessment of pesticide exposure}

Assessment of exposure is considered as the main issue when dealing with epidemiological evidence on pesticides available so far, due to intrinsic difficulties in characterizing exposure to individual active substances. Pesticide exposure can be modelled by using validated questionnaires or job-exposure matrices, although biomonitoring can provide better metrics of

\footnotetext{
${ }^{4}$ https://echa.europa.eu/documents/10162/23715527/msc-57 minutes en.pdf/11f11007-1814-48fc-5818$\underline{775 f 9 e 12 \mathrm{e} 8 \mathrm{ec} .}$
} 
exposure. "Exposome" approaches and molecular epidemiology open new possibilities for research and advanced risk assessment bridging toxicology and epidemiology.

The exposome, that is, the totality of exposures received by an individual during life time represents a challenging but promising new concept in the field and currently there are tools available to measure exposome, e.g. biomarkers of the internal exposome (xenobiotics and metabolites), or the use of -omic technologies (adductome, metabolome, transcriptome, epigenome, proteome) (Vineis et al., 2017), although still only for research purposes.

Developments in the field of molecular epidemiology will improve exposure assessment, document early changes in the toxicity pathway preceding disease, and identify subgroups in the population with greater susceptibility to adverse outcomes. Thereby, the ability of epidemiological studies to identify causal risk factors and elucidate mechanisms underlying pathogenesis of diseases will increase. The implementation of molecular epidemiology tools, especially connected with exposome, AOPs/MOAs and systems toxicology, will provide additional possibilities for exposure assessment and health risk prediction.

It is important to consider what pesticide exposure actually means in the context of epidemiology research and experimental research. In real life, long-term exposures are almost always complex regarding both PPP and other chemical exposures (either simultaneously or sequentially), whereas in experimental studies exposures are mostly to single pesticide active substances and at high doses, which represents an unrealistic scenario. This dilemma creates problems when risk assessments of combined exposure to multiple chemicals are performed.

\section{The use of AOP as a scaffold to provide biological plausibility to epidemiological findings}

The AOP framework is a useful tool for risk assessment to explore whether an adverse outcome is biologically plausible or not. By mechanistically substantiating apical endpoints or outcomes, the AOP contributes to the inclusion of human data in hazard identification and characterization steps in risk assessment. Thus, AOPs allow moving towards a mechanisticbased risk assessment.

If strong epidemiological evidence is available, there is no need to use an AOP for going ahead with risk assessment. However, even in this case, an AOP can still provide additional support on a positive finding, especially on the identification of potential risk factors (lifestyles, genetics, environmental chemicals, etc.) identified by the intermediate key events. Where epidemiological studies of specific diseases (e.g. PD) would be time-consuming and expensive, and often would identify individual pesticides or groups of pesticide, an AOP 
would provide insight into their risk factors. This can be particularly useful for chronic human degenerative diseases where gene-environmental interactions strongly influence the risk, severity and progression of such diseases and where the ability of animal model of replicate the disease associated pathology is very limited..

In these cases, AOPs are built starting from adverse outcomes, thus matching a hazard profile of a specific exposure (chemical/stressor) interacting with a molecular initiating event (MIE) and triggering the linear chain of key events eventually leading to the AO. However, it seems unlikely that a single AOP can explain all endpoints of complex diseases.

In cases where modest or weak associations between adverse outcomes and exposures are found, AOPs would provide supportive evidence for the mechanistic biological plausibility or, contrary, negative evidence for the pathogenesis of a disease.

\section{Quality assessment of human epidemiological studies}

Quality in epidemiological studies represents an issue for individual studies, which covers from study design to study reporting, and for pooled evidence.

Key factors to determine whether epidemiology findings should be taken into account for a WoE assessment are addressed by assessing the risk of bias for observational epidemiological studies based on specific tools available (US-EPA, 2016; EFSA PPR Panel, 2017b). If this assessment is part of the evidence synthesis where epidemiological research is assessed and quantitatively summarised, it permits more accurate estimation of the magnitude of the effect related to pesticide exposure. This is an important point as pesticide risk assessment should not be based on results of epidemiological studies that do not meet well-defined data quality standards, because a high risk of bias challenges the internal validity of a study.

When a systematic review is conducted to synthesize evidence, assessment of methodological quality and risk of bias of the selected studies should be performed. Individual studies should be evaluated for possible selection bias, measurement error, sampling error, heterogeneity, study design, and reporting and presentation of results. In addition, meta-analysis allows for examining additional bias, such as small study effects, publication bias and excess significance bias.

\section{Training issue as a necessary enabling factor}

Experts from different disciplines are needed for the balanced integrated risk assessment of pesticides. There exists a consensus about expertise needed for the evaluation of 
epidemiological evidence in risk assessment of pesticides. Ideally, epidemiologists trained in (chemical) risk assessment are required as well as a permanent dialogue between epidemiologists and toxicologists (and many other disciplinary experts at least occasionally if needed, such as experts in exposure science).

Specific case studies on the use of epidemiological evidence for pesticide risk assessment would be valuable especially for training purposes. US-EPA has some examples: Dicamba for Tier I, 2,4-D and Permethrin for Tier II and Atrazine, Glyphosate and Chlorpyrifos for Tier III.

\section{Future Guidance from EFSA Scientific Committee}

Because this opinion piece present EFSA experiences, it is proper to finalize with some recommendations to EFSA: although there are also diverse views, it seems preferable that an overarching Guidance should be drafted by the EFSA Scientific Committee regarding chemicals in general, not only pesticides (this activity is foreseen ).

Exposure assessment should be the prime consideration and investment to be made when dealing with the EFSA guidance, because it is the most obvious gap in knowledge creating uncertainty. However, for some chemicals, such as heavy metals, smoking, alcohol, or organochlorine compounds, exposure can be properly characterized, although also these exposures involve usually other simultaneously exposing multiple chemicals.

Vigilance observations, including medical data, are an under-sought source of information for chemical risk assessment. A section in the future guidance for the use of such data should be developed.

\section{Conclusions}

Risk assessment of pesticides is a complex task. Besides, regulatory toxicity tests may do not fully address adverse effects observed in human epidemiological studies. On the other hand, these may not be sensitive enough or focused to ever detect significant harmful effects. Consequently, the following proposals would enhance a more human-relevant and hazardtargeted risk assessment of pesticides:

- The use of the AOP conceptual framework to provide the mechanistic basis for a biological plausible link between a MIE and an AO found in epidemiological studies.

- An initial framework for the evaluation and integration of epidemiological observations in the pesticides risk assessment. 
- Since the most obvious gap in the proposed approach is the complexity of performing an adequate exposure assessment, this should be overcome by implementing human biomonitoring, - omic technologies, or exposome analysis, which takes a holistic approach by combining data from multiple sources.

- A guidance to facilitate the risk assessment process of chemicals in general by using a multidisciplinary approach. 


\section{References}

Agricultural Health Study (AHS). https://aghealth.nih.gov/

Andreotti G, Koutros S, Hofmann JN, Sandler DP, Lubin JH, Lynch CF, Lerro CC, De Roos AJ, Parks CG, Alavanja MC, Silverman DT, Beane Freeman LE (2018) Glyphosate Use and Cancer Incidence in the Agricultural Health Study. J Natl Cancer Inst 110:509-516.

Bal-Price A, Meek MEB (2017) Adverse outcome pathways: Application to enhance mechanistic understanding of neurotoxicity. Pharmacol Ther 179:84-95. doi: 10.1016/j.pharmthera.2017.05.006.

Bal-Price A, Lein PJ, Keil KP, Sethi S, Shafer T, Barenys M, Fritsche E, Sachana M, Meek ME (2017) Developing and applying the adverse outcome pathway concept for understanding and predicting neurotoxicity. Neurotoxicology 59:240-255. doi: 10.1016/j.neuro.2016.05.010.

Bhat VS, Meek MEB, Valcke M, English C, Boobis A, Brown R (2017) Evolution of chemical-specific adjustment factors (CSAF) based on recent international experience; increasing utility and facilitating regulatory acceptance. Crit Rev Toxicol 47(9):729-749. doi: 10.1080/10408444.2017.1303818.

Choi J, Polcher A, Joas A (2016) Systematic literature review on Parkinson's disease and Childhood Leukaemia and mode of actions for pesticides. Supporting Publications 2016:EN -955. 256 pp. Available online: www.efsa.europa.eu/publication

Delrue N, Sachana M, Sakuratani Y, Gourmelon A, Leinala E, Diderich R (2016) The adverse outcome pathway concept: A basis for developing regulatory decision-making tools. Altern Lab Anim 44(5):417-429.

Edwards SW, Tan YM, Villeneuve DL, Meek ME, McQueen CA (2016) Adverse Outcome Pathways-Organizing Toxicological Information to Improve Decision Making. J Pharmacol Exp Ther 356(1):170-81. doi: 10.1124/jpet.115.228239.

EFSA PPR Panel (EFSA Panel on Plant Protection Products and their Residues), Ockleford C, Adriaanse P, Berny P, Brock T, Duquesne S, Grilli S, Hernandez-Jerez AF, Bennekou SH, Klein M, Kuhl T, Laskowski R, Machera K, Pelkonen O, Pieper S, Smith R, Stemmer M, Sundh I, Teodorovic I, Tiktak A, Topping CJ, Wolterink G, Angeli K, Fritsche E, Hernandez-Jerez AF, Leist M, Mantovani A, Menendez P, Pelkonen O, Price A, Viviani B, Chiusolo A, Ruffo F, Terron A and Bennekou SH (2017a) Scientific Opinion on the 
investigation into experimental toxicological properties of plant protection products having

EFSA PPR Panel (EFSA Panel on Plant Protection Products and their Residues), Colin Ockleford, Paulien Adriaanse, Philippe Berny, Theodorus Brock, Sabine Duquesne, Sandro Grilli, Susanne Hougaard, Michael Klein, Thomas Kuhl, Ryszard Laskowski, Kyriaki Machera, Olavi Pelkonen, Silvia Pieper, Rob Smith, Michael Stemmer, Ingvar Sundh, Ivana Teodorovic, Aaldrik Tiktak, Chris J. Topping, Gerrit Wolterink, Matteo Bottai, Thohallur Halldorsson, Paul Hamey, Marie-Odile Rambourg, Ioanna Tzoulaki, Daniele Court Marques, Federica Crivellente, Hubert Deluyker and Antonio F. Hernandez-Jerez (2017b) Scientific Opinion of the PPR Panel on the follow-up of the findings of the External Scientific Report "Literature review of epidemiological studies linking exposure to pesticides and health effects". EFSA Journal 2017b; 15(10):5007, 101 pp. https://doi.org/10.2903/j.efsa.2017.5007

Fritsche E, Crofton KM, Hernandez AF, Hougaard Bennekou S, Leist M, Bal-Price A, Reaves E, Wilks MF, Terron A, Solecki R, Sachana M, Gourmelon A (2017) OECD/EFSA workshop on developmental neurotoxicity (DNT): The use of non-animal test methods for regulatory purposes. ALTEX 34(2):311-315. doi: 10.14573/altex.1701171.

Goodman M, Naiman DQ, LaKind JS (2018) Systematic review of the literature on triclosan and health outcomes in humans. Crit Rev Toxicol 48(1):1-51. doi: 10.1080/10408444.2017.1350138.

Hernandez AF, Tsatsakis AM (2017) Human exposure to chemical mixtures: Challenges for the integration of toxicology with epidemiology data in risk assessment. Food Chem Toxicol 103:188-193. doi: 10.1016/j.fct.2017.03.012.

Hernandez AF, Menendez P (2016) Linking Pesticide Exposure with Pediatric Leukemia: Potential Underlying Mechanisms. Int J Mol Sci 17(4):461. doi: 10.3390/ijms17040461. LaKind JS, Sobus JR, Goodman M, Barr DB, Först P, Albertini RJ, Arbuckle TE, Schoeters G, Tan YM, Teeguarden J, Tornero-Velez R, Weisel CP (2014) A proposal for assessing study quality: Biomonitoring, Environmental Epidemiology, and Short-lived Chemicals (BEES-C) instrument. Environ Int 73:195-207. doi: 10.1016/j.envint.2014.07.011.

LaKind JA, Idri F, Naiman DQ, Verner MA (2019) Biomonitoring and Nonpersistent Chemicals - Understanding and Addressing Variability and Exposure Misclassification. Current Environmental Health Reports https://doi.org/10.1007/s40572-019-0227-2 
Meek ME, Palermo CM, Bachman AN, North CM, Jeffrey Lewis R (2014) Mode of action human relevance (species concordance) framework: Evolution of the Bradford Hill considerations and comparative analysis of weight of evidence. J Appl Toxicol 34(6):595-606. doi: 10.1002/jat.2984.

Moher D, Shamseer L, Clarke M, Ghersi D, Liberati A, Petticrew M, Shekelle P, Stewart LA, PRISMA-P Group (2015) Preferred reporting items for systematic review and metaanalysis protocols (PRISMA-P) 2015 statement. Syst Rev 2015 Jan 1;4:1. doi:10.1186/2046-4053-4-1.

Ntzani EE, Chondrogiorgi M, Ntritsos G, Evangelou E, Tzoulaki I (2013) Literature review on epidemiological studies linking exposure to pesticides and health effects. EFSA supporting publication 2013:EN-497, 159 pp.

Pelkonen O, Terron A, Hernandez AF, Menendez P, Bennekou SH, EFSA WG EPI1 Members (2017) Chemical exposure and infant leukaemia: development of an adverse outcome pathway (AOP) for aetiology and risk assessment research. Arch Toxicol 91(8): 2763-2780. doi: 10.1007/s00204-017-1986-X

Rhomberg LR, Goodman JE, Bailey LA, Prueitt RL, Beck NB, Bevan C, Honeycutt M, Kaminski NE, Paoli G, Pottenger LH, Scherer RW, Wise KC, Becker RA (2013) A survey of frameworks for best practices in weight-of-evidence analyses. Crit Rev Toxicol 43(9):753-84. doi: 10.3109/10408444.2013.832727.

Sakuratani Y, Horie M, Leinala E (2018) Integrated Approaches to Testing and Assessment: OECD Activities on the Development and Use of Adverse Outcome Pathways and Case Studies. Basic Clin Pharmacol Toxicol 123 Suppl5:20-28. doi: 10.1111/bcpt.12955.

SAPEA, Science Advice for Policy by European Academies (2018) Improving authorisation processes for plant protection products in Europe: a scientific perspective on the potential risks to human health. Berlin: SAPEA. https://doi.org/10.26356/plantprotectionproducts

Scientific Advice Mechanism (SAM) (2018) EU authorisation processes of Plant Protection Products from a scientific point of view. ISBN 978-92-79-67735-9 doi:10.2777/238919 KI-04-17-354-EN-N.

Shamseer L, Moher D, Clarke M, Ghersi D, Liberati A, Petticrew M, Shekelle P, Stewart LA; PRISMA-P Group (2015) Preferred reporting items for systematic review and metaanalysis protocols (PRISMA-P) 2015: elaboration and explanation. BMJ 2015 Jan 2;350:g7647. doi: 10.1136/bmj.g7647. 
Terron A, Bal-Price A, Paini A, Monnet-Tschudi F, Bennekou SH; EFSA WG EPI1

Members, Leist M, Schildknecht S (2018) An adverse outcome pathway for parkinsonian motor deficits associated with mitochondrial complex I inhibition. Arch Toxicol 92(1):4182. doi: 10.1007/s00204-017-2133-4.

US-EPA (United States Environmental Protection Agency) (2016) Office of Pesticide Programs' Framework for Incorporating Human Epidemiologic \& Incident Data in Risk Assessments for Pesticides December 28, 2016 https://www3.epa.gov/pesticides/EPA-HQOPP-2008-0316-DRAFT-0075.pdf

Vineis P, Chadeau-Hyam M, Gmuender H, Gulliver J, Herceg Z, Kleinjans J, Kogevinas M, Kyrtopoulos S, Nieuwenhuijsen M, Phillips DH, Probst-Hensch N, Scalbert A, Vermeulen R, Wild CP (2017) EXPOsOMICS Consortium. The exposome in practice: Design of the EXPOsOMICS project. Int J Hyg Environ Health 220:142-151.

Internet sources used in the article

Adverse outcome pathway knowledge base (AOP-KB): http://aopkb.org/

AOP-wiki: https://aopwiki.org/ 
Legend for Figure 1. The project landscape of the EFSA scientific opinions on Parkinson's disease and childhood leukaemia and on integration of epidemiology and experimental research. Time frame (upper part), epidemiological studies (middle part) and AOP framework (lower part) schematically presented.

EFSA activities in relation to pesticide epidemiology

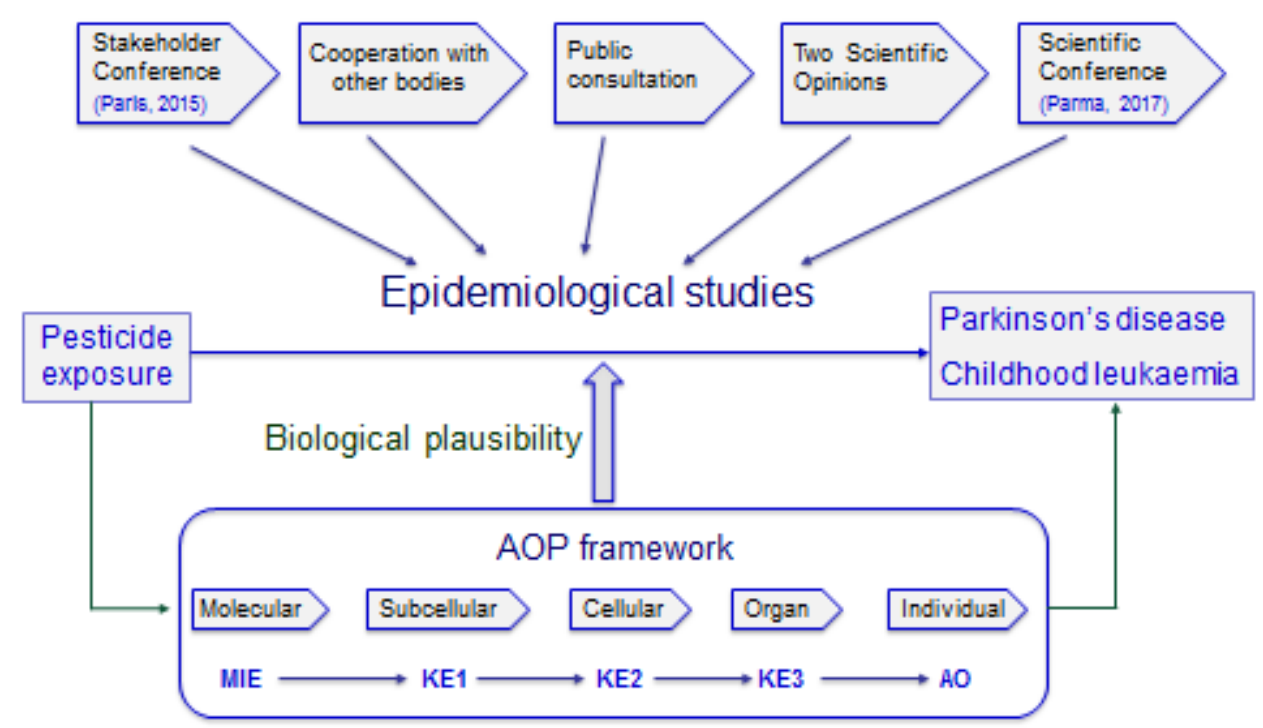


Legend for Figure 2. Use of epidemiological evidence for pesticide risk assessment: strengths and limitations

Risk assessment

Biological plausibility?

\section{Epidemiological studies}

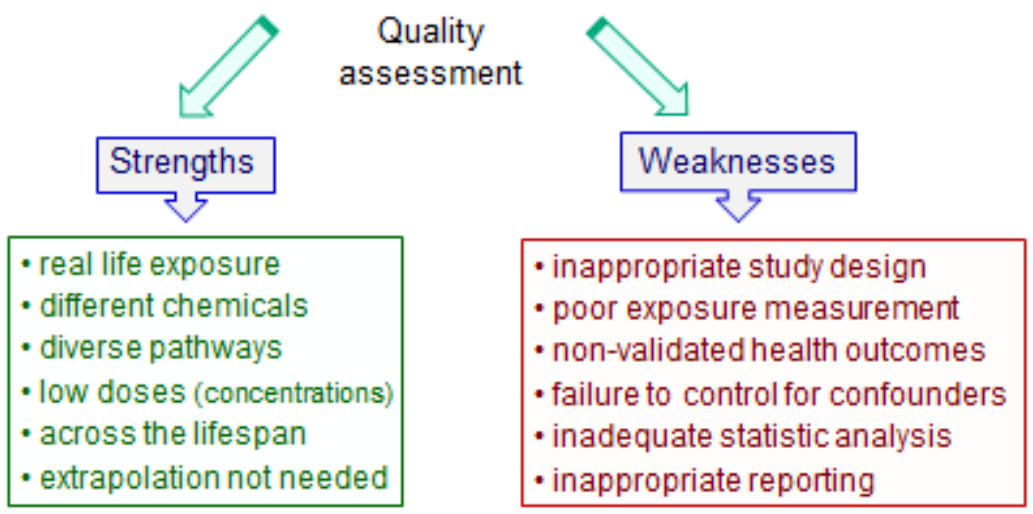

